\title{
Radar Signal Emitter Recognition Based on Combined Ensemble Empirical Mode Decomposition and the Generalized S-Transform
}

\author{
Bin Liu $\left(\mathbb{D},{ }^{1}\right.$ Youqian Feng, ${ }^{2}$ Zhonghai Yin, ${ }^{2}$ and Xiangyu Fan ${ }^{1}$ \\ ${ }^{1}$ Graduate School, Air Force Engineering University, Xian 710051, China \\ ${ }^{2}$ Department of Basic Sciences, Air Force Engineering University, Xian 710051, China
}

Correspondence should be addressed to Bin Liu; 277306879@qq.com

Received 8 January 2019; Accepted 20 March 2019; Published 21 April 2019

Academic Editor: Gilberto Espinosa-Paredes

Copyright (C) 2019 Bin Liu et al. This is an open access article distributed under the Creative Commons Attribution License, which permits unrestricted use, distribution, and reproduction in any medium, provided the original work is properly cited.

\begin{abstract}
Present radar signal emitter recognition approaches suffer from a dependency on prior information. Moreover, modern emitter recognition must meet the challenges associated with low probability of intercept technology and other obscuration methodologies based on complex signal modulation and must simultaneously provide a relatively strong ability for extracting weak signals under low SNR values. Therefore, the present article proposes an emitter recognition approach that combines ensemble empirical mode decomposition (EEMD) with the generalized S-transform (GST) for promoting enhanced recognition ability for radar signals with complex modulation under low signal-to-noise ratios in the absence of prior information. The results of Monte Carlo simulations conducted using various mixed signals with additive Gaussian white noise are reported. The results verify that EEMD suppresses the occurrence of mode mixing commonly observed using standard empirical mode decomposition. In addition, EEMD is shown to extract meaningful signal features even under low SNR values, which demonstrates its ability to suppress noise. Finally, EEMDGST is demonstrated to provide an obviously better time-frequency focusing property than that of either the standard S-transform or the short-time Fourier transform.
\end{abstract}

\section{Introduction}

RADAR signal emitter recognition is an important aspect of electronic warfare reconnaissance systems that seeks to identify individual radar emitters through an analysis of the electromagnetic signals and thereby determine vital information regarding the technical level, performance, position, and deployment conditions of enemy radar systems for supporting decision making regarding enemy weapon systems and targets [1]. However, radar emitters must be accurately identified under complex and highly variable electromagnetic environments to ensure the military significance of emitter recognition [2-4].

The radar signal emitter recognition problem has been the subject of intensive investigation, and substantial development has been achieved. For example, an improved evidence modeling method that sought to fuse multisensor information was proposed to improve the accuracy of radar emitter recognition $[5,6]$. However, the method requires expert experience or the support of extensive sample data in calculating entropy and hyperentropy, and this heavy reliance on prior information limits the applicability of the method. Moreover, the performance of the method depends on the quantity of signal detected and received, which is itself strongly related to fusion times, and this commonly results in a lack of related evidence that further limits the applicability of the method, particularly with the recent rapid development of low probability of intercept (LPI) radar signals. Furthermore, a matching pursuit method that functioned under a semisupervised mode was proposed to realize atom refactoring in a redundant dictionary for obtaining optimum linear combinations [7]. However, constructing the redundant dictionary requires a level of prior information, and the computational time required for seeking linear combinations in the dictionary must be improved. In addition, an emitter signal model was constructed using knowledgebased statistical signal processing with a syntactic domain knowledge representation to recognize radar emitters $[8$, 
9]. However, the method requires sufficient and correct prior information to accurately identify a radar emitter, which is relatively difficult to obtain under modern warfare conditions. A radar emitter recognition method has also been proposed, which adopts genetic engineering theory, particularly reverse engineering, as a reference for conducting emitter recognition [10]. However, the method presently resides in a qualitative description phase and is not sufficiently developed to support practical applications. A radar emitter recognition method has also been proposed, which expresses emitter signals in random changing forms between multiple modes according to different pulse reoccurrence intervals [11]. However, modern LPI radar signals adopt highrate random fluctuations between multiple modes, which significantly complicates the task of obtaining solid signal patterns at any given moment. Radar emitters have also been recognized by methods employing a secondary treatment level of data, for example, by constructing pulse sample figures based on vague sets [12] and by constructing an emitter recognition model with reference to rough set theory $[13,14]$. While these methods possess the advantages of easy operation and realization, they also suffer from the loss of weak signal features, which makes it difficult to conduct fine recognition.

As discussed, the development of LPI technology has become an increasing challenge for electronic reconnaissance because LPI reduces the probability of signal interception by the passive reconnaissance equipment of an opposing party by controlling the transmitted power, signal bandwidth, signal parameters, and modulation patter. In addition, modern radar emitter signals adopt various modulation types to reduce the probability of being intercepted, making it difficult for radar interception receivers to effectively recognize signal features within a single dimension of time or frequency. Therefore, the development of emitter recognition methods must meet the challenges associated with the present and future development of LPI technology and other obscuration methodologies. Simultaneously, the prevalence of electromagnetic signals in electromagnetic space has undergone a sharp increase in modern times, which leads to both intentional and unintentional interference effects. Although the intensity of intentional interference is low, its effect is precisely calculated. Meanwhile, the sources and intensities of unintentional interference vary greatly, and this has the effect of decreasing the signal-to-noise ratio (SNR), which correspondingly increases the difficulty of signal detection. Therefore, modern radar reconnaissance interception receivers are required to provide a relatively strong ability for extracting weak signals under low SNR values.

From the above analysis of past work and existing challenges, we note that present radar emitter recognition approaches suffer most profoundly from subjective intervention and a severe dependency on prior information. Therefore, the present study eliminates this dependency on prior information by combining ensemble empirical mode decomposition (EEMD) with the generalized S-transform (GST) to realize the recognition of radar signal emitters. EEMD can be used to conduct adaptive extraction of intrinsic mode functions (IMFs) on each scale component of emitter signals from high frequency to low frequency with good local transient characteristic representation capacity and good noise suppression. The GST is then applied to the IMFs obtained by decomposition, and the time-frequency planar features of signals are characterized and extracted using the good time-frequency focusing property of GST for realizing effective radar signal emitter recognition. These advantages of the proposed EEMD-GST approach are verified by Monte Carlo simulations conducted using various mixed signals with additive Gaussian white noise.

\section{Basic Theory}

2.1. Empirical Mode Decomposition. Empirical mode decomposition (EMD) decomposes a signal $x(t)$ over some arbitrary range of time $t$ into components denoted as IMFs that must have the following two properties [15]:

(1) The number of extrema and the number of times the function crosses zero are either equal or differ by only 1 at most.

(2) The two envelopes formed by the function maxima and function minima must be symmetric about the $t$ axis (i.e., the difference between the maximum and minimum envelopes must be zero).

The specific sifting process by which $x(t)$ is decomposed into IMFs is illustrated in Figure 1 and discussed as follows $[16,17]$.

Step 1. Determine all extrema of $x(t)$ over an arbitrary range of $t$. Interpolate all maxima using a cubic spline function to form the upper envelope, and apply the same method to the minima to form the lower envelope. The mean value of the upper and lower envelopes of $x(t)$ is defined as $m_{1}$, and the first component $h_{1}$ of $x(t)$ is defined as follows.

$$
h_{1}(t)=x(t)-m_{1}
$$

Step 2. Decomposition is further conducted by treating $h_{1}$ as if it were $x(t)$, and step 1 is repeated $i$ times until $h_{i}$ meets the two conditions of an IMF.

Step 3. The first IMF obtained from $x(t)$ represents the shortest-period mode of the signal and is designated as $c_{1}$. This IMF is then separated from $x(t)$ as follows.

$$
r_{1}=x(t)-c_{1}
$$

Step 4. Decomposition is further conducted by treating $r_{1}$ as if it were $x(t)$ and repeating steps 1 and $2 n$ times until no further IMF components can be obtained. The remaining signal

$$
r_{n}=r_{n-1}-c_{n}
$$

is a monotonic function denoted as the remnant, representing the average trend of $x(t)$.

Step 5. The full EMD representation of $x(t)$ is then given as follows.

$$
x(t)=\sum_{j=1}^{n} c_{j}(t)+r_{n}
$$




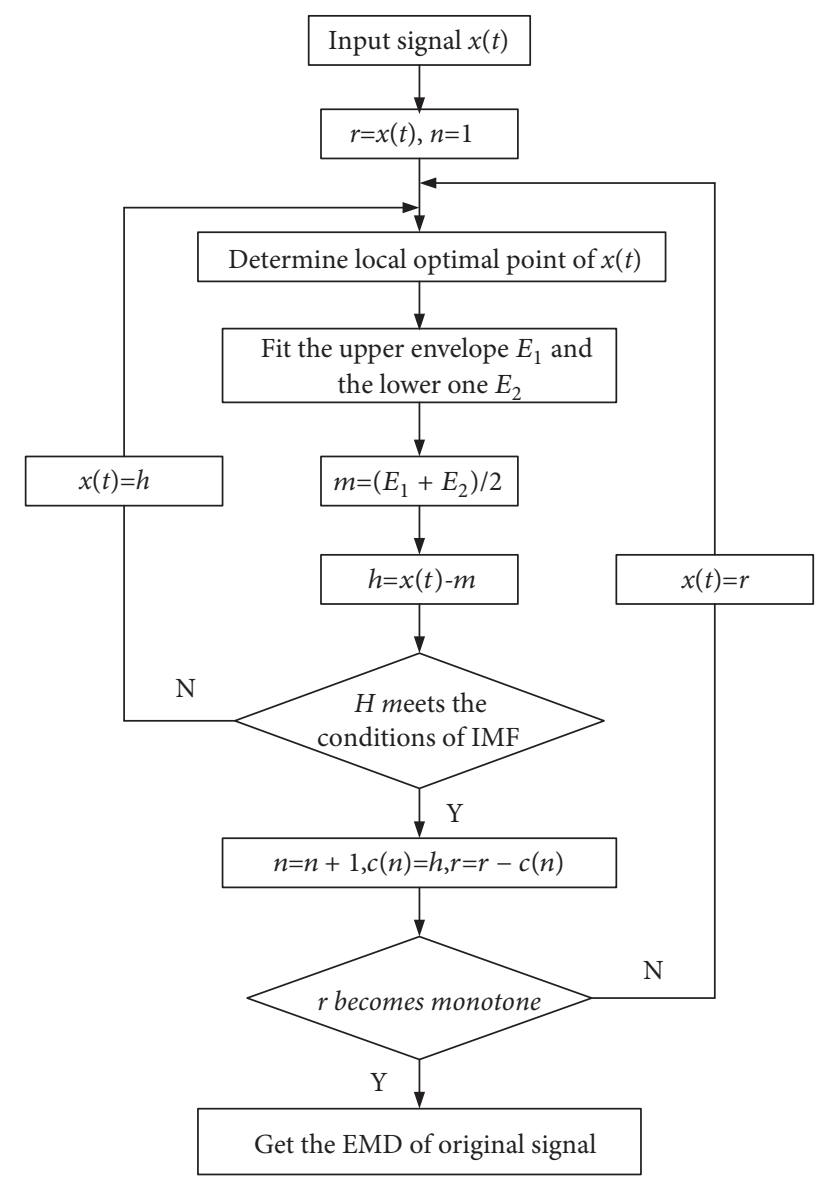

FIGURE 1: Basic process flow of empirical mode decomposition (EMD).

According to the sifting process, each IMF component $c_{j}$ represents different periodic modes from high to low. Moreover, the instantaneous frequency of IMF at different moments changes along with changes in the signal itself.

We note from the above process that EMD can be applied to any signal, requires no prior information or assumptions, and can decompose signals into different modes adaptively according to the signal features itself. However, the application of EMD to a signal whose time scale changes rapidly can induce mode mixing, where a signal feature operative over widely different time scales is decomposed into a single IMF, or a signal feature operative over very similar time scales can be included in different IMFs. However, mode mixing limits the performance of EMD severely $[18,19]$.

2.2. Ensemble Empirical Mode Decomposition. The mode mixing problem associated with EMD has been addressed by EEMD. In EEMD, an IMF is defined as the average IMF obtained over an ensemble of $N$ trials using EMD, where each decomposition is conducted for the original signal $x(t)$ plus additive Gaussian white noise (AWGN) of some amplitude that is varied randomly for each trial $[20,21]$. The EEMD process is illustrated in Figure 2 and discussed as follows [22].
The following are the abbreviated implementation steps of the proposed algorithm.

Step 1. Initialize the number of evaluations $N$.

Step 2. Set evaluation index $i=1$.

Step 3. Add white noise $n_{i}(t)$ with a unique amplitude uncorrelated with any past amplitudes to $x(t)$ to produce the modified signal.

$$
x_{i}(t)=x(t)+n_{i}(t)
$$

Step 4. Decompose $x_{i}(t)$ by EMD to obtain $j=1,2, \ldots, n$ IMFs. The full EMD representation of $x_{i}(t)$ of the $i$ th added white noise sequence is then given as follows.

$$
x_{i}(t)=\sum_{j=1}^{n} \mathrm{IMF}_{i j}+r_{i j}
$$

Step 5. Repeat steps 3 and $4 N$ times while appropriately incrementing $i$ to obtain the set of IMFs:

$$
\left[\left\{\mathrm{IMF}_{1 j}\right\},\left\{\mathrm{IMF}_{2 j}\right\}, \cdots,\left\{\mathrm{IMF}_{N j}\right\}\right]
$$

where $j=1,2, \ldots, n$.

Step 6. Based on the principle that the statistical average of uncorrelated sequences is zero, the mean values of the ensemble IMFs in (7) are calculated to obtain the final IMFs.

$$
\mathrm{IMF}_{j}=\frac{1}{N} \sum_{i=1}^{N} \mathrm{IMF}_{i j}
$$

Step 7. The final result of EEMD decomposition is

$$
x(t)=\sum_{j=1}^{n} \mathrm{IMF}_{j}+\sum_{j=1}^{n} r_{j}
$$

where $r(t)$ is the residual component.

The decomposition times of EEMD are different due to different signals, noise intensity, and the number of layers. However, for the final result of EEMD decomposition, most of them only have a set of maximum and minimum values, so an attempt can be made to determine the decomposition layers of EEMD $[23,24]$.

2.3. Generalized S-Transform. The S-transform adopts the Fourier kernel and a frequency dependent window function of variable window length to obtain higher frequency resolution at lower frequencies and better time localization at higher frequencies [25]. Thus, conceptually, the S-transform can be considered a hybrid of short-time Fourier analysis and wavelet analysis. The short-time Fourier transform (STFT) of a signal $x(t)$ can be defined as

$$
s(t, f)=\int_{-\infty}^{\infty} x(\tau) w(t-\tau) e^{-j 2 \pi f \tau} d \tau
$$




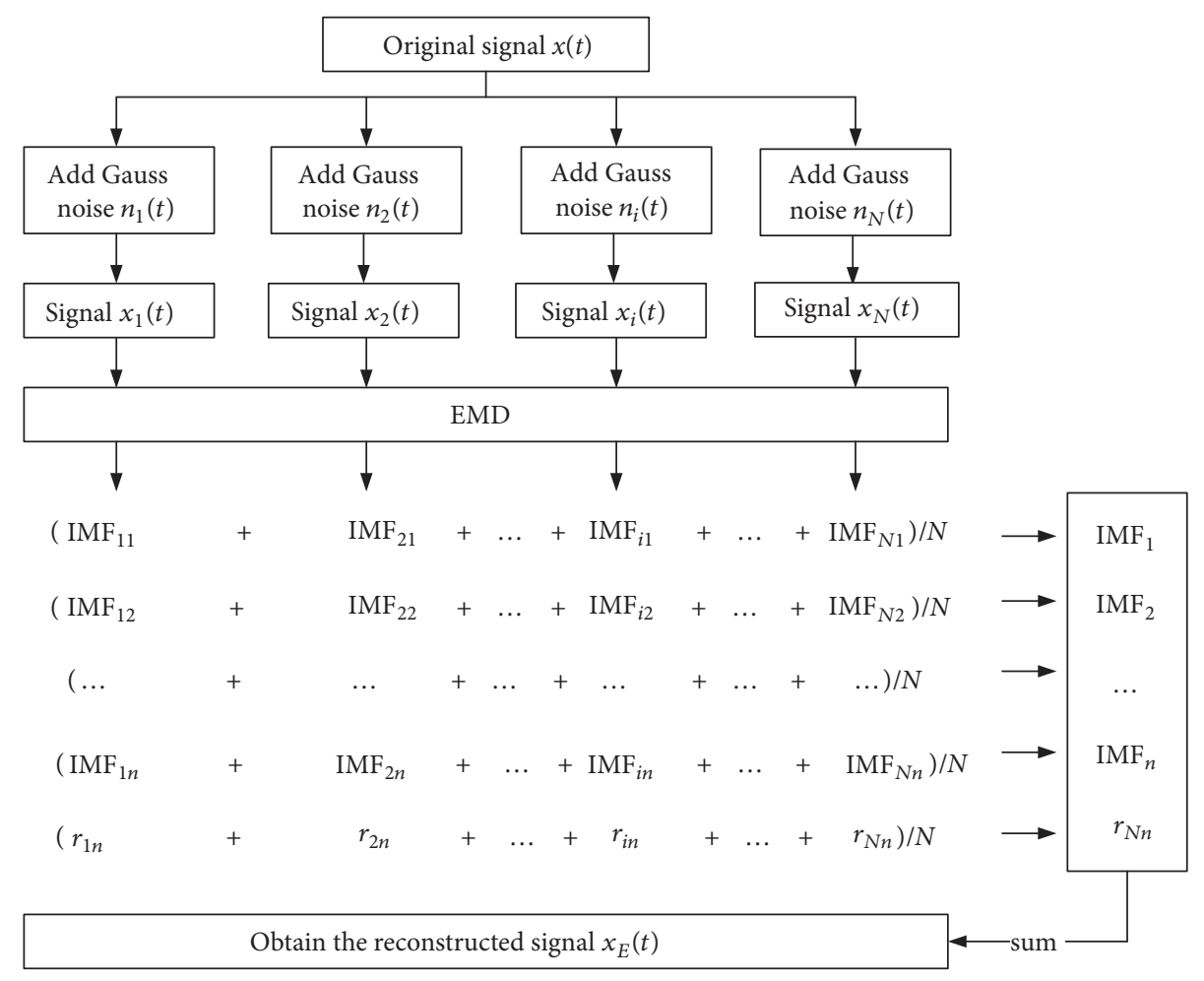

FIGURE 2: Basic process flow of ensemble EMD (EEMD).

where $\tau$ is a time-shift factor, $f$ is the frequency, $w(t)$ is a window function, and $j=\sqrt{ }-1$. Here, $w(t)$ is defined as a normalized Gaussian window:

$$
w(t)=\frac{1}{\sigma \sqrt{2 \pi}} e^{-t^{2} / 2 \sigma^{2}}
$$

where $\sigma=1 / f$ is the scale factor of the Gaussian window. After substituting $\sigma=1 / f$ into (11), and (11) into (10), the Stransform of $x(t)$ can be obtained as follows.

$$
s(t, f)=\int_{-\infty}^{\infty} x(\tau) \frac{|f|}{\sqrt{2 \pi}} e^{-(t-\tau)^{2} f^{2} / 2} e^{-j 2 \pi f \tau} d \tau
$$

From this expression, we note that the inverse relationship of $\sigma$ to $f$ provides a frequency resolution capacity for signals that increases with decreasing $f$, and a temporal resolution for signals that increases with increasing $f$.

Presently, primary airborne battle radar mainly adopts the $\mathrm{X}$-band to obtain better high-frequency resolution, so as to realize effective detection in passive reconnaissance activities. Therefore, to enhance the time-frequency resolution capacity of the S-transform and thus improve its timefrequency focusing property, the GST applies a regulation coefficient $p$ as a power law coefficient to $\sigma$ as follows.

$$
\sigma=\frac{1}{|f|^{p}}
$$

Here, (12) can be rewritten when $p>0$ to obtain the GST of $x(t)$ as follows.

$$
\operatorname{GST}(t, f)=\int_{-\infty}^{\infty} x(\tau) \frac{|f|^{p}}{\sqrt{2 \pi}} e^{-(t-\tau)^{2} f^{2 p} / 2 k^{2}} e^{-j 2 \pi f \tau} d \tau
$$

Accordingly, the determination of $p$ directly affects the time-frequency resolution capacity of the GST, and the value of the parameter must therefore be carefully optimized. The present study adopts a previously published optimization criterion for $p$ [26], where the objective is to minimize the energy concentration measure $M(p)$ of the GST timefrequency representation of $x(t)$ for $p \in(0,1]$. This is defined as follows.

$$
M(p)=\left(\sum \sum|\operatorname{GST}(t, f)|^{1 / p}\right)^{p}
$$

In the above formula, $\sum \sum \mathrm{GST}(t, f)$ serves as the coefficient of energy normalization. According to above the criterion, the steps for optimizing $p$ are illustrated as follows [27].

Step 1. For an arbitrary $p \in(0,1]$, apply the GST to $x(t)$ using (14).

Step 2. Apply normalization processing to the GST.

$$
\operatorname{NGST}(t, f)=\frac{\operatorname{GST}(t, f)}{\sum \sum \mid \operatorname{GST}(t, f)}
$$


Step 3. Select $p=2$, and calculate $M(p)$ using (15).

Step 4. Repeat steps 1, 2, and 3 until obtaining the optimal value of $p$, denoted as $p_{o p t}$, that provides a minimum $M(p)$.

$$
p_{o p t}=\arg \min _{p}(M(p))
$$

Finally, an intelligent search algorithm can be employed in the optimization process, and the optimum GST is obtained by substituting $p_{o p t}$ into (14).

\section{Radar Signal Recognition Process Based on EEMD-GST}

At present, the radar is developing toward LPI. By controlling its transmitting power, signal bandwidth, signal parameters, and modulation styles, it can realize effective detection while reducing the probability of being intercepted by the enemy's passive detection equipment, thus improving the ability of radar to perform tasks. Therefore, the development of LPI technology has become an urgent challenge for electronic reconnaissance.

In order to improve the recognition efficiency of radar signals without prior information under strong noise interference, based on the discussion in the previous section, this paper constructs the radar signal recognition process based on EEMD-GST. It is mainly divided into three parts, namely, signal preprocessing, system parameter adjustment, and signal recognition. The specific steps involved in the proposed EEMD-GST radar signal emitter recognition approach are given as follows.

3.1. Signal Preprocessing. Although it is assumed that the received radar signal is in the $\mathrm{X}$-band, the $\mathrm{X}$-band has a bandwidth of $4 \mathrm{G}$, and the modulation style and amplitude of the signal are completely unknown. If the received signal is processed directly, the result of processing is likely not ideal under many unknown conditions, and the parameter interpretation is likely to be inaccurate. Therefore, in this paper, the signal is first obtained and interpreted through the EEMD-GST detection process.

The specific steps are as follows.

Step 1. Discrete the signals obtained by the reconnaissance interception receiver, and input them into the EEMD-GST signal processing system.

Step 2. Apply EEMD to decompose the time series signals, and obtain the IMFs.

Step 3. Apply Particle Swarm Optimization algorithm to obtain the optimal GSTs of IMFs.

Step 4. Analyze the time-frequency distribution figure obtained to evaluate the features of intercepted signals.

Step 5. Combine signal characteristics based on the analysis of GSTs for each IMF to realize radar signal emitter recognition.
Step 6. Parameterize the signal according to the signal recognition results, and simulate the detection signal approximately, namely, the signal to be detected and SNR to be estimated.

3.2. Parameters Adjusting of the System. In the process of EEMD processing, the total average number $N$ is highly correlated with the algorithm efficiency. The larger the value of $N$ is, the weaker the mode aliasing phenomenon will be, and the influence of noise will be weakened. At the same time, due to the excessive average number of times, the subtle features of the signal will gradually weaken or even disappear with the increase of $N$, thus affecting the recognition of signal modulation and even the interpretation of parameters. On the contrary, with the decrease of $N$, the impact of noise will be intensified and even generate mode aliasing, resulting in recognition errors. At the same time, for different signals or even the same signal under different SNRs, the values of $N$ may be different. Therefore, it is necessary to adjust the value of $N$ by combining the characteristics of signal with noise to cancel the mode aliasing and preserve the subtle features of signal.

In the GST transformation process, the adjustment factor $p$ also affects the algorithm efficiency. Although the timefrequency analysis method can directly show the relationship between the signal time and frequency, the contradiction between the resolution and frequency resolution time always restricts the time-frequency analysis method. There is theoretical limit of the product of two resolutions. In general, for a certain time and frequency processing method, the improvement of one resolution is at the cost of degradation of another resolution, and GST is no exception. However, $p$ has two functions. The first one is to dynamically adjust one resolution according to the demand, and the other one, as shown in (15), is to improve the focusing ability of the time-frequency distribution. In other words, the product of two resolutions approximates to the theoretical limit, and the two resolutions can be simultaneously promoted. Similarly, different signals may have different values of $p$. It is necessary to adjust the value of $p$ adaptively according to the signal parameters so as to improve the signal detection ability.

Therefore, this section builds an adaptive optimization process with two parameters, which is shown in Figure 4.

Step 1. Construct the simulated signal $s_{0}(t)$ according to the result of signal preprocessing, and mix it with noise, so that the SNR turns to SNRe.

Step 2. Set the average number $N$ of EEMD.

Step 3. Decompose the mixed signal by EEMD to obtain the $N * n$ IMF matrix, and sum the signal to obtain the reconstructed signal $s_{r}(t)$.

Step 4. Calculate the peak signal-to-noise ratio (PSNR) and noise intensity $D$ of the reconstructed signal $s_{r}(t)$. Then calculate the maximum PSNR (denoted as PSNR $\operatorname{Pax}_{\text {max }}$ ) of $s_{0}(t)$ under the noise background with intensity $D$. 
Step 5. Determine whether the current PSNR is greater than $0.9 * \mathrm{PSNR}_{\max }$, which is to judge whether most of the characteristics of the signal are retained. If the conditions are met, proceed to the next step. Otherwise, adjust the parameter $N$ according to the control law and return to Step 2.

Step 6. Set the adjustment factor $p$ of GST.

Step 7. Conduct GST to the reconstructed signal to obtain the time-frequency distribution diagram. Calculate the timefrequency distribution diagram of the simulated signal $s_{0}(t)$ in the noise background with intensity $D$, and analyze the structural similarity (SSIM) of the two diagrams.

Step 8. Determine whether SSIM is greater than 0.9 , which is to find whether the signals before and after processing are similar and whether the details are well retained. When SSIM is greater than 0.9 , most information of the signal is considered to be retained and we can proceed to Step 9 . Otherwise, adjust $p$ and return to Step 6 .

Step 9. Complete the parameter setting of the system.

In the above process, calculating the PSNR and SSIM can be completed by directly calling MATLAB function package, which is easy to implement. Next we will introduce the way to adjust $N$, namely, the control law of $N$ :

$$
\begin{aligned}
\operatorname{err}(k) & =P S N R_{\max }-P S N R(k) \\
u(k) & =w \times \operatorname{err}(k) \\
N(k+1) & =N(k)+u(k)
\end{aligned}
$$

where $k$ in (18) denotes the $k$ th adjustment, and $\operatorname{err}(k)$ represents the difference between $\operatorname{PSNR}(k)$ and the limit PSNR $_{\max }$, which will be used as the error feedback. $u(k)$ is the proportional control variable, and $w$ is the proportional coefficient, which equals 100 in the subsequent simulation. Equation (18) provides the recurrence equation to adjust the average number $\mathrm{N}$ of the EEMD, so as to realize the adjustment of the $N$ and achieve the optimal matching of parameters. Equation (18) is a typical error feedback proportional control, which is simple, stable, and easy to realize.

The control method of parameter $p$ is similar to (18), as shown in (19).

$$
\begin{aligned}
\operatorname{err}_{p}(k) & =0.9-\operatorname{SSIM}(k) \\
u_{p}(k) & =w_{p} \times \operatorname{err}_{p}(k) \\
p(k+1) & =p(k)+u_{p}(k)
\end{aligned}
$$

Many parameters are similar to (18). We take 2 in the subsequent simulation, and the other parameters are not described again. In this way, the adjustment of parameter $p$ is achieved.

To sum up, the system parameters can be adjusted dynamically and adaptively according to different signal

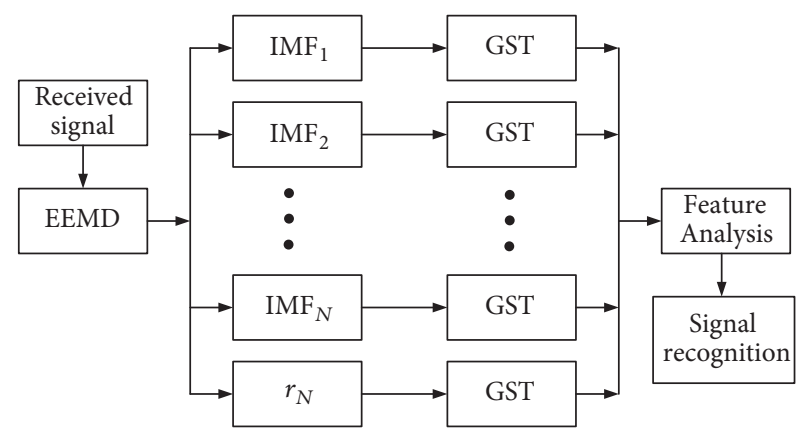

FIGURE 3: Radar signal emitter recognition process based on EEMD and the generalized S-transform (GST).

parameters and modulation styles, so as to achieve the approximate matching between system parameters and the signal to be detected.

3.3. Signal Recognition. By constructing the approximated parameter matching system and inputting the signal to be detected to the processing flow in Figure 3, the interpretation of signal modulation style and parameters can be realized.

As there is no a priori information of the signal to be detected, if the system parameter is fixed during the detection procedure, the generality of test may be poor and the result may not be ideal. Therefore, the proposed flow preprocesses the detection signal to obtain approximated prior information. A negative feedback mechanism and a corresponding control law are designed, and the system parameters are dynamically adjusted to match the signals to be detected as much as possible. Finally, the signal to be detected is input into the processing flow with adjusted parameters to realize identification, thereby greatly improving the accuracy of signal recognition. The applicability and universality of the algorithm are stronger, and the performance is further improved

\section{Simulation Experiments}

Monte Carlo simulation analyses were conducted to demonstrate the advantages of the proposed EEMD-GST radar signal emitter recognition approach from three different perspectives. Firstly, EMD and EEMD were applied to decompose a mixed signal formed as a linear superposition of a high-frequency intermittent signal and a simple continuous signal, and the decomposition results were compared to demonstrate that EEMD can effectively suppress mode mixing. Secondly, EEMD was applied to decompose mixed signals composed of a standard linear frequency modulated (LFM) signal and additive Gaussian white noise of different amplitudes. Then, the ability of EEMD to extract meaningful signal features was evaluated under different SNR values to demonstrate the ability of the algorithm to suppress noise. Thirdly, EEMD-GST processing was applied to a mixed signal involving the linear superposition of several typical radar signals with additive white Gaussian noise, and the analysis results obtained were compared with the corresponding 


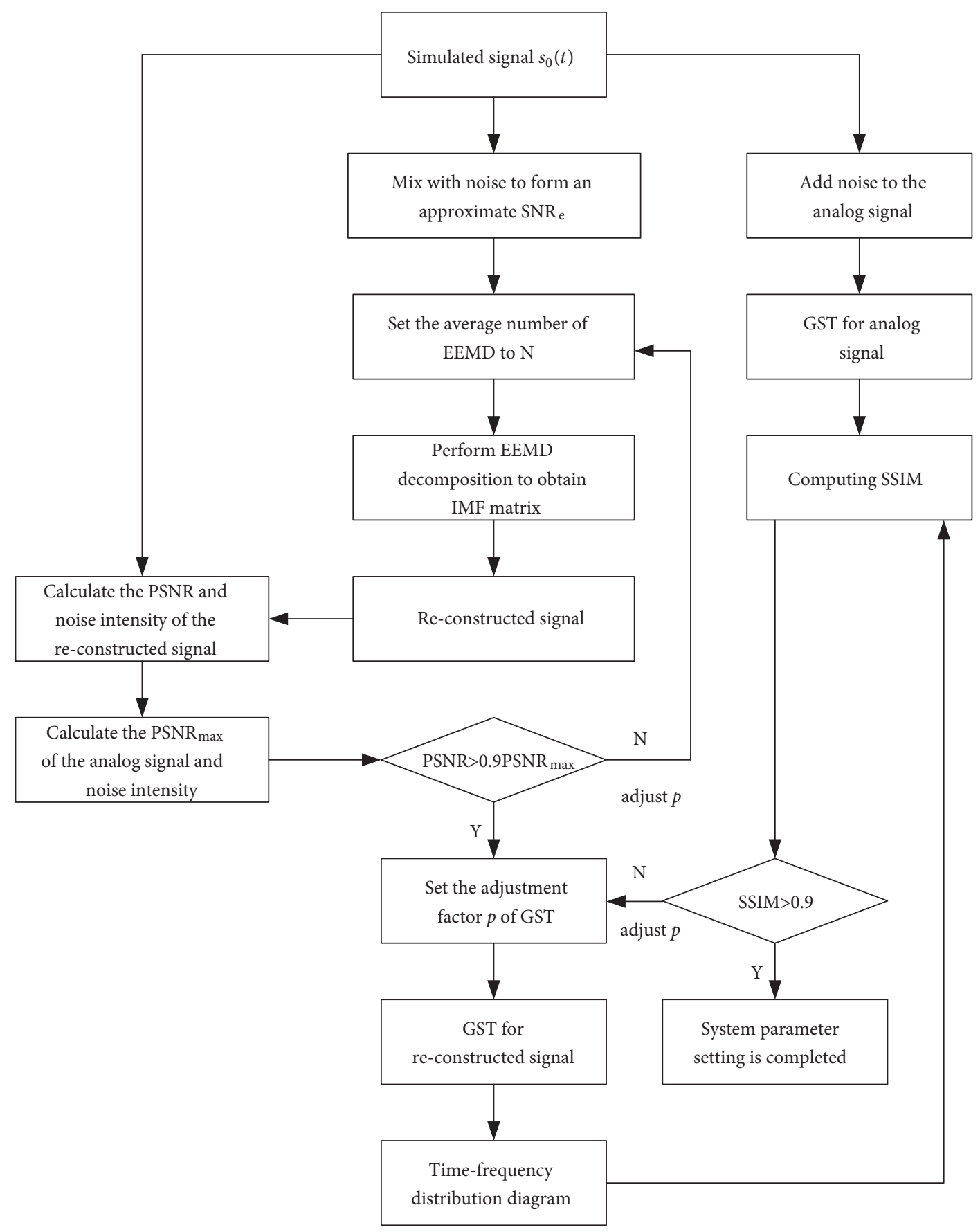

FIgURE 4: Processing flow of algorithm parameters adjustment.

results obtained using the standard S-transform (ST) and the short-time Fourier transform (STFT) to verify the effectiveness of the proposed approach. The simulations employed 5 sets of Lenovo personal computers in parallel with Core ${ }^{\mathrm{TM}}$ i7$3770 \mathrm{CPUs}$ operating under a clock frequency of $3.40 \mathrm{GHz}$ and $4 \mathrm{~GB}$ of memory.

Electronic reconnaissance is highly covert. Often using optical camouflage, rarely moving and with ground clutter coverage, passive detection equipment is extremely difficult to detect by opposing radar. At the same time, the electronic reconnaissance party itself is passive, and it is impossible for the enemy passive detection equipment to detect our passive reconnaissance equipment. Similar to how many sound sources can be detected in a dark environment, it is difficult to tell how many listeners there are. As a result, it is impossible to determine how many passive reconnaissance devices are in the battlefield, and it is more difficult to determine the location of equipment and other information. As a result, it is impossible to conduct precise spoofing interference on passive equipment. The interference faced 

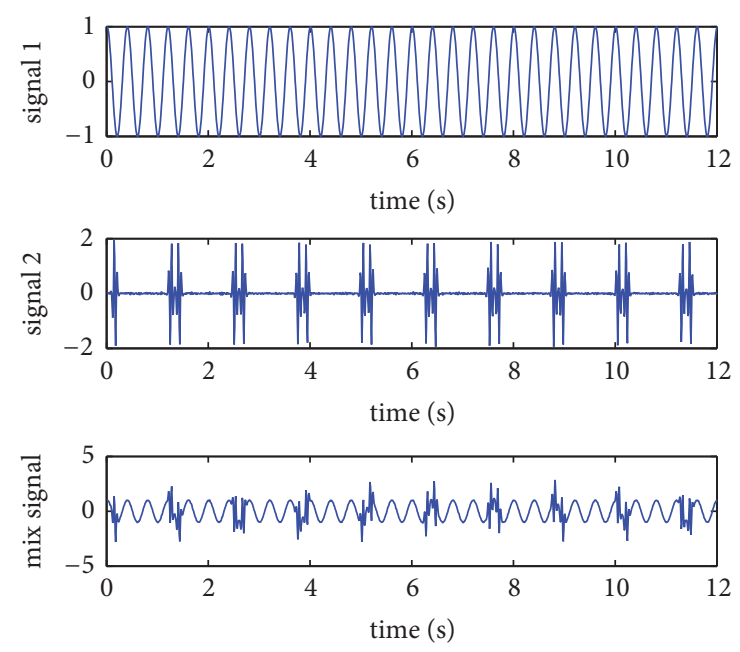

FIGURE 5: Linear superposition of a low-frequency cosine function signal with steady-state characteristics (signal 1) and a burst-type signal with high-frequency transient components (signal 2).

by passive equipment is mainly strong noise. Therefore, in order to be closer to the reality, the signal-to-noise ratio is set lower in the subsequent simulation verification to reflect the applicability of the method.

4.1. Comparison of EMD and EEMD Mode Isolation Performances. Standard radar signals generally represent a pulse pattern, particularly for airborne fire-control radar. As such, radar silence is generally maintained while approaching an objective, and radar is suddenly deployed only when within close proximity to an objective, resulting in signal patterns characterized by strong intermittency. Therefore, the decomposition results of EMD and EEMD were compared for the mixed signal shown in Figure 5 composed of a lowfrequency cosine function signal with steady-state characteristics (signal 1) and a burst-type signal with high-frequency transient components (signal 2). A signal time length of $12 \mathrm{~s}$ and a sampling frequency of $1 \mathrm{kHz}$ were adopted. The mixed signal was decomposed by EMD and EEMD, where EEMD adopted a range of AWGN amplitudes that was 0.5 times that of the mixed signal. The average time of the signal was 20, and Monte Carlo experiments were conducted 100 times. The averages of the IMF results obtained by EMD and EEMD are shown in Figures 6(a) and 6(b), respectively.

Comparing Figure 5 with Figure 6(a), we note that severe mode mixing appears in the IMFs obtained through EMD due to the transient high-frequency components in the mixed signal. As a result, each IMF obtained loses its physical significance, and even false IMFs are obtained. These results clearly illustrate the limitations of EMD for radar signal emitter recognition applications. However, comparing Figures 5 and 6(b), we note that all IMFs obtained by EEMD represent the characteristics of the original signal to some extent and can effectively reflect its physical characteristics, which clearly illustrates the better processing effect of EEMD relatively to that of EMD. Simultaneously, we also note that the components IMF2 and IMF5 obtained by EEMD are very similar to the respective signals 1 and 2 shown in Figure 5, as would be expected for the decomposed components of a multicomponent signal.

Therefore, to further quantify and measure algorithm performance, we adopted the similarity coefficient $\xi_{i j}$ of a source signal time series $s_{i}^{\prime}(t)$ composed of $k$ elements and a separated signal time series $s_{j}(t)$ as an index for measuring the separation effect of EED and EEMD. This index is defined as follows.

$$
\xi_{i j}=\xi\left(s_{i}^{\prime}, s_{j}\right)=\left|\frac{\sum_{t=1}^{k} s_{i}^{\prime}(t) s_{j}(t)}{\sqrt{\sum_{t=1}^{k} s_{i}^{\prime 2}(t) \sum_{t=1}^{k} s_{j}^{2}(t)}}\right|
$$

The value of $\xi_{i j}$ calculated between IMF5 obtained via EEMD and signal 1 was 0.9901, and that between IMF2 and signal 2 was 0.9476 . However, the largest value of $\xi_{i j}$ calculated between any IMF obtained via EMD and signal 1 was 0.8533 , and that with signal 2 was only 0.5279 . Therefore, we can conclude that, compared with EMD, EEMD not only can suppress mode mixing to some extent, but also can obtain better decomposability, which contributes toward realizing the effective preprocessing of radar signals.

4.2. Analysis of EMD and EEMD Noise Suppression Performances. The antinoise capability of EEMD was evaluated in comparison to that of EMD when applied to a typical LFM signal with additive Gaussian white noise of different intensities. The assumed simulated signal is given as

$$
x(t)=s(t)+n(t)
$$

where $n(t)$ is additive Gaussian white noise of arbitrary amplitude and $s(t)$ is a common LFM signal employed in radar systems.

$$
s(t)=\sin [2 \pi(50+20 t) t]
$$

A signal time length of $1 \mathrm{~s}$ and a sampling frequency of $10 \mathrm{kHz}$ were adopted. The amplitude range of the additive Gaussian white noise employed in EEMD was 0.3 times that of the mixed signal. The average time of the signal was 20 , and the range of the SNR was $[-20,5] \mathrm{dB}$. To ensure the effectiveness of the simulation results, Monte Carlo experiments were conducted 200 times under conditions of different SNR values and 5,200 times in total. The decomposition performances of EMD and EEMD were quantitatively evaluated by calculating the largest value of $\xi_{i j}$ using formula (20) and the lowest mean squared error (MSE) with the original signal for all IMFs obtained from decomposition under different SNR values. The $\xi_{i j}$ and MSE results are presented in Figures 7(a) and 7(b), respectively.

We can directly note from Figure 7(a) that the largest values of $\xi_{i j}$ for the IMFs obtained by EEMD are always greater than those obtained by EMD, and the IMFs obtained by EEMD maintain a largest $\xi_{i j}$ value of approximately 0.2 for an SNR of $-20 \mathrm{~dB}$. Therefore, EEMD provides a better processing effect than EMD under very low SNR. Moreover, the IMFs obtained by EEMD maintain a largest $\xi_{i j}$ value of 


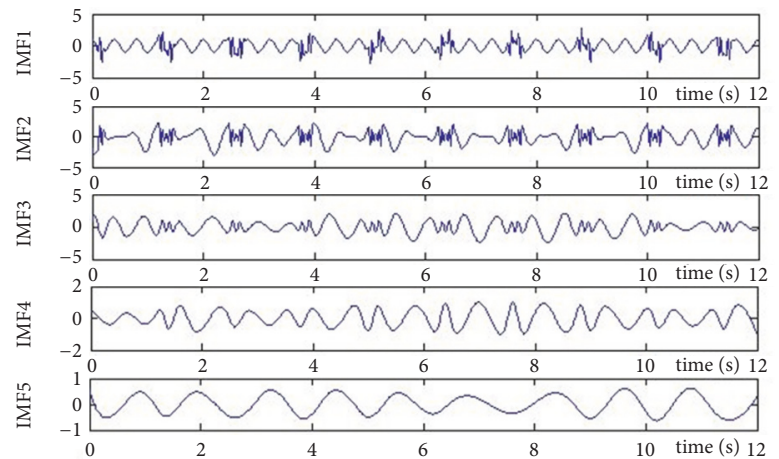

(a) EMD decomposition result

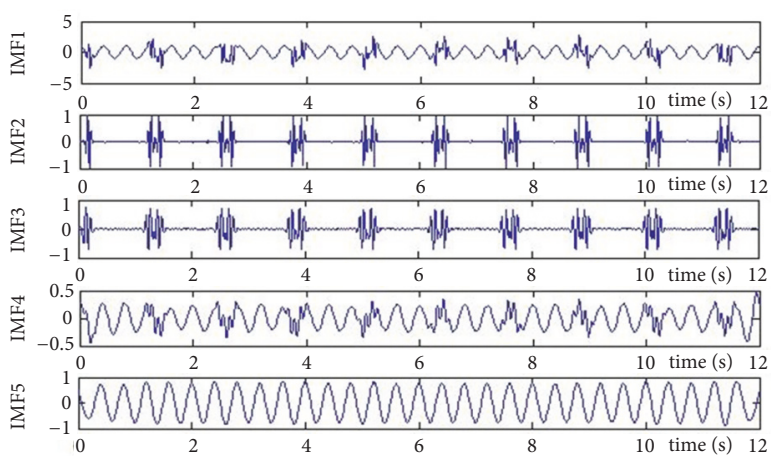

(b) EEMD decomposition result

FIGURE 6: Comparison of the decomposition results of (a) EMD and (b) EEMD for the mixed signal given in Figure 4.

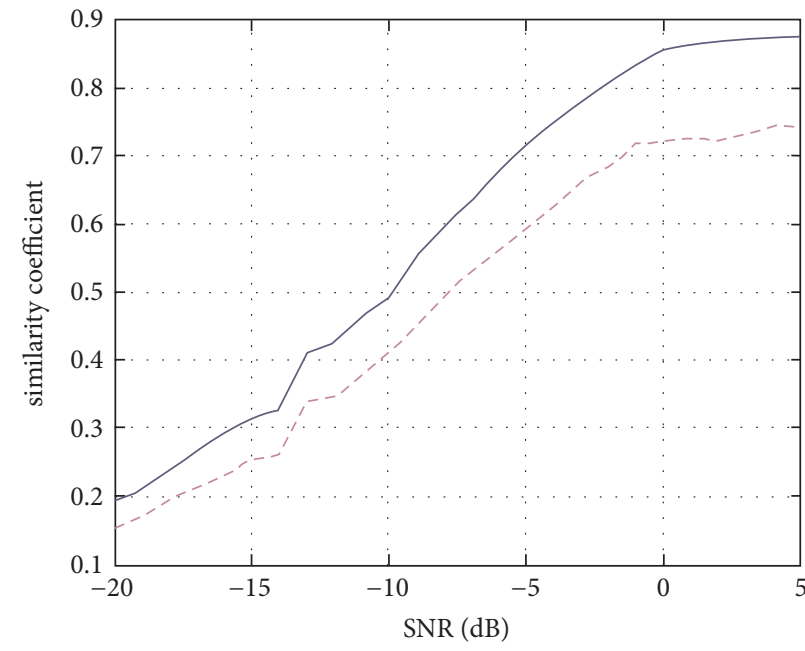

EMD

- EEMD

(a) Comparison diagram of the highest similarity coefficient under different signal-to-noise ratios

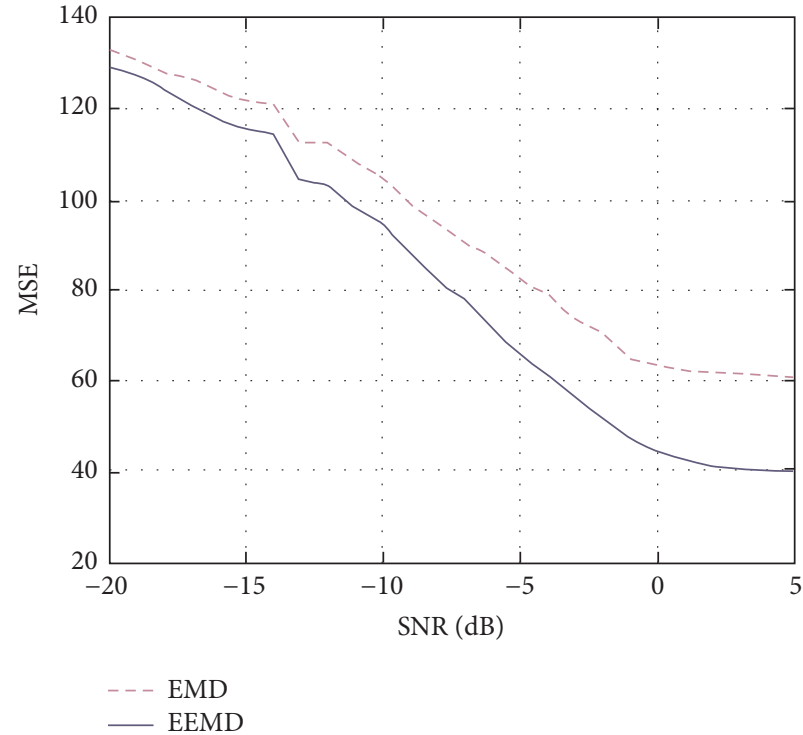

(b) Comparison diagram of the lowest MSE under different signal-tonoise ratios

FIGURE 7: Comparison of (a) the similarity coefficient and (b) mean squared error (MSE).

approximately 0.5 at an $\mathrm{SNR}$ of $-10 \mathrm{~dB}$, which represents a good representation for signal recognition. In addition, we note from Figure 7(b) that the MSE values obtained by EEMD through a large number of simulation experiments are always less than those obtained by EMD. Moreover, the differences in the MSE values obtained by EEMD and EMD continuously increase with increasing SNR up to the later stage when the MSE values stabilize, and the stable MSE value obtained by EEMD is obviously less than the stable value obtained by EMD.

These results are a clear reflection of the processing conducted by EMD and EEMD, as discussed in Section 2. Here, the single decomposition conducted by EMD under a strong noise interference background largely fails to suppress the noise interference, and the characteristics of the actual signal are correspondingly obscured. Meanwhile, EEMD conducts decomposition numerous times under a randomly fluctuating additive Gaussian white noise, and the randomness of the noise makes it theoretically possible to decompose each IMF equally based on the premise that a large number of trials increases the probability of the noise being evenly distributed, such that adopting the average value will weaken the influence of noise. Simultaneously, the actual signal components are generally decomposed into equivalent IMFs, or a high degree of the actual signal information will be included within a particular IMF. Thus, the information pertaining to the actual signal is accumulated continuously with additional decomposition, which progressively increases the $\xi_{i j}$ values obtained between the IMFs and the actual signal, thus suppressing the influence of noise.

The above simulation results and theoretical analysis demonstrate that EEMD can be applied for the extraction 


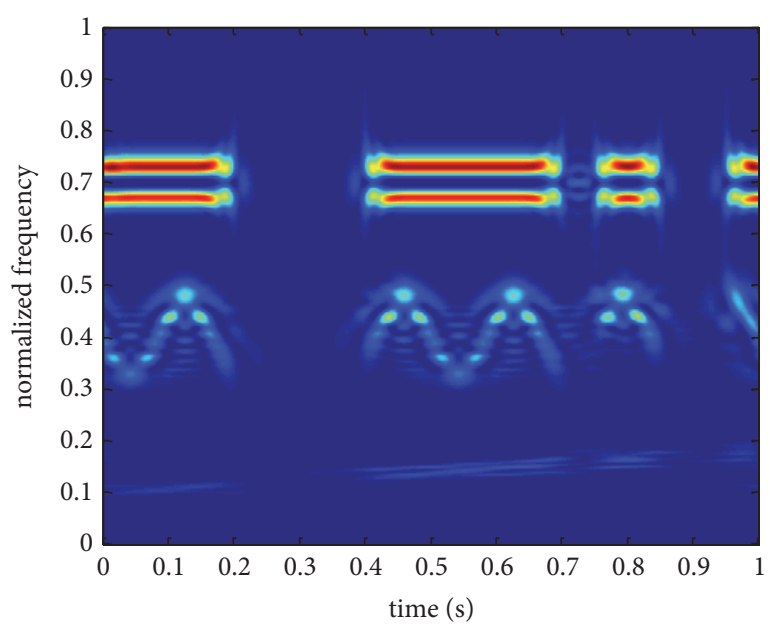

(a) EEMD-GST time-frequency transform diagram

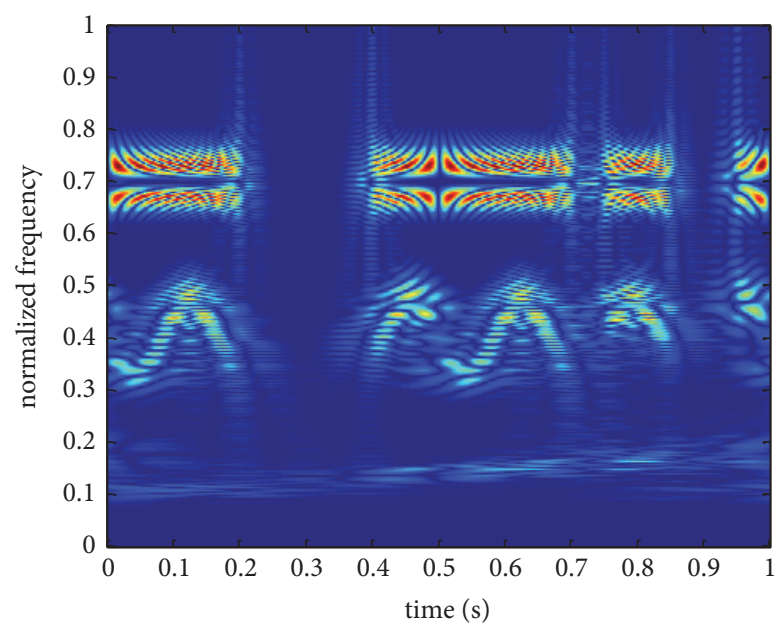

(b) ST time-frequency transform diagram

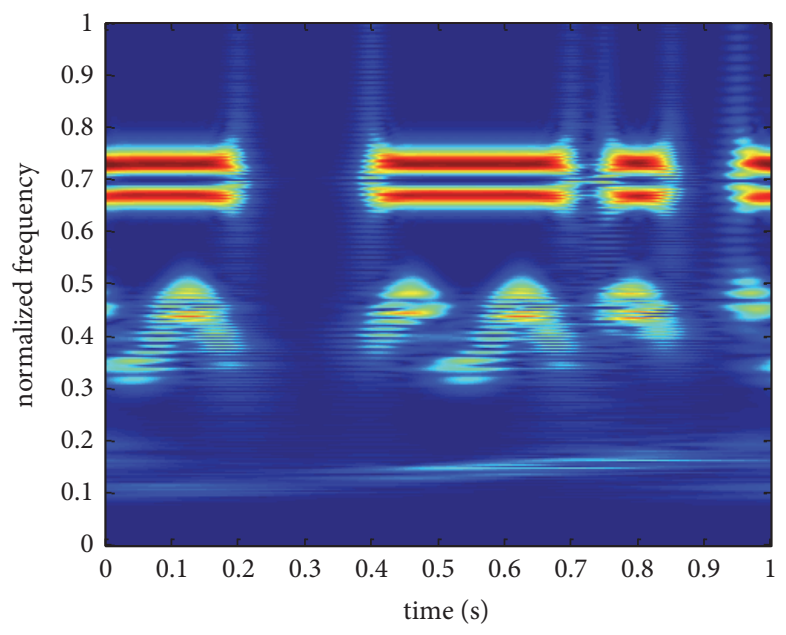

(c) STFT time-frequency transform diagram

FIgURE 8: Time-frequency analysis results.

of weak signals under a strong noise background with good processing effect, and this can be expected to aid in the detection and analysis of LPI signals under low SNR values.

4.3. Radar Signal Emitter Recognition Based on EEMDGST. To verify the effectiveness of the proposed EEMDGST approach, we constructed a linear superposition of the following four typical harmonic signals.

$$
\begin{aligned}
& s_{1}(t)=\sin [2 \pi(50+20 t) t] \\
& s_{2}(t)=\sin [2 \pi 200 t+2 \pi \cos (12 \pi t)] \\
& s_{3}(t)=\sin (2 \pi 300 t) \\
& s_{4}(t)=\cos (2 \pi 350 t)
\end{aligned}
$$

Here, $s_{1}$ is an LFM signal, $s_{2}$ is a phase-modulated signal, and $s_{3}$ and $s_{4}$ are sinusoidal signals. As such, we defined the actual mixed signal as $s(t)=s_{1}(t)+s_{2}(t)+s_{3}(t)+s_{4}(t)$. A signal time length of $1 \mathrm{~s}$ and a sampling frequency of $10 \mathrm{kHz}$ were adopted.
First, the mixed signal $s(t)$ with no additive Gaussian white noise was processed by EEMD-GST, while abandoning the weak part of the IMFs and the remainder term. In addition, processing was conducted by the ST and STFT using an equivalent simulation platform as that employed in the previous subsection. The time-frequency distribution results for EEMD-GST, ST, and STFT are shown in Figures 8(a), 8(b), and $8(\mathrm{c})$, respectively. The four signal components from the bottom to the top of each figure are, respectively $s_{1}, s_{2}, s_{3}$, and $s_{4}$. We can note directly from the figures that the simulation result obtained by EEMD-GST presents an obviously better time-frequency focusing property than that of either ST or STFT. Although the ST characterizes the features of the phase-modulated signal $s_{2}$ reasonably well, its resolution for sinusoidal signals $s_{3}$ and $s_{4}$ remains relatively poor. However, the STFT cannot clearly characterize the time-frequency characteristics of LFM signal $s_{1}$ and phase-modulated signal $s_{2}$. As such, we can quantitatively conclude that EEMD-GST can provide the time-frequency characteristics required for emitter recognition. 


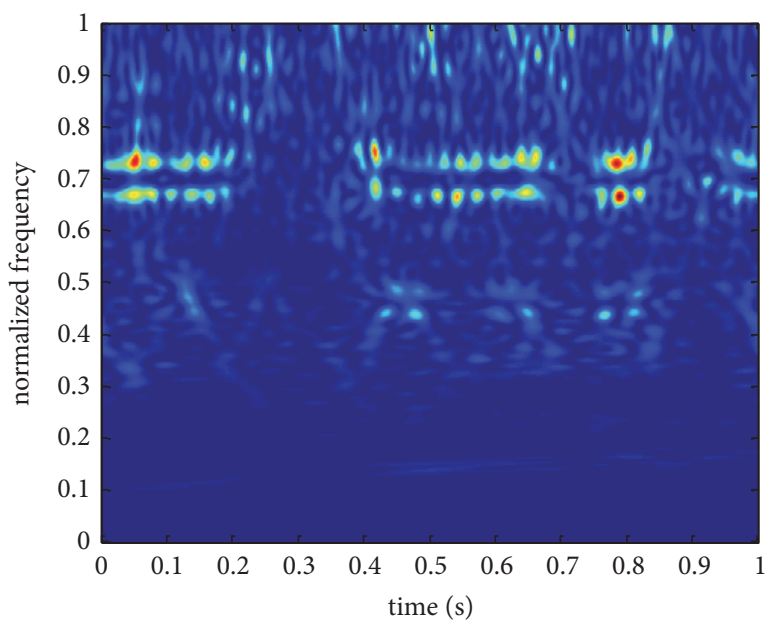

(a) EEMD-GST time-frequency transform diagram under - $12 \mathrm{~dB}$

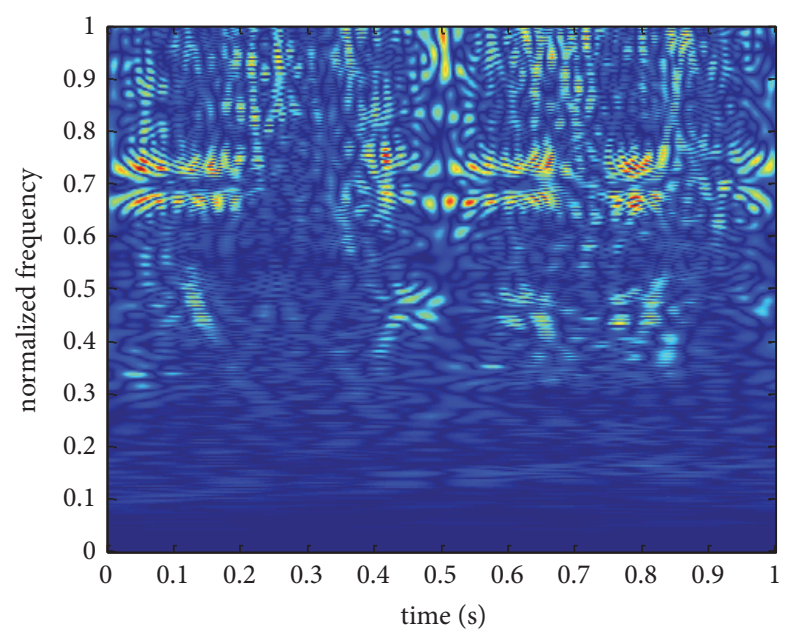

(b) ST time-frequency transform diagram under $-12 \mathrm{~dB}$

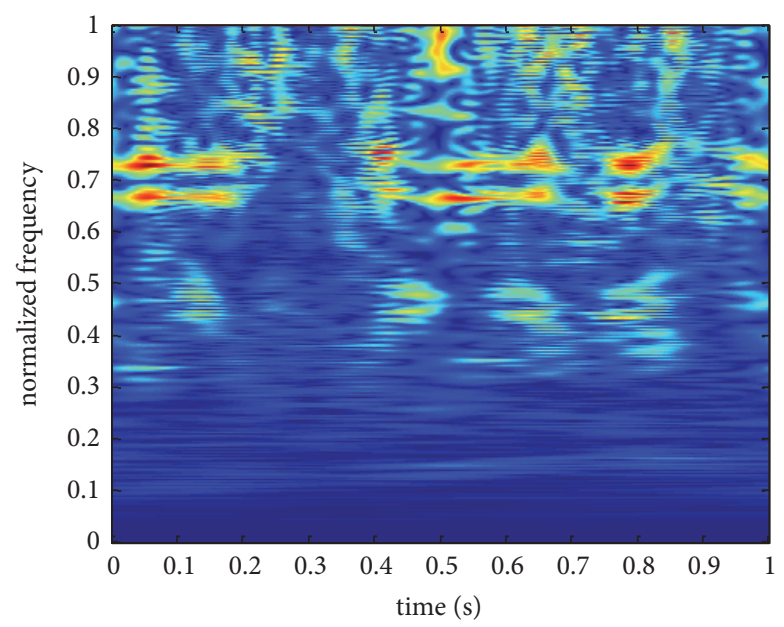

(c) STFT time-frequency transform diagram under $-12 \mathrm{~dB}$

FIGURE 9: Time-frequency analysis results obtained by (a) EEMD-GST, (b) ST, and (c) STFT under an SNR of -12 dB.

At the same time, in order to ensure that the signal may be of burst type, after intercepting a section of signal, the time-frequency analysis is carried out on it, and the results are shown in Figure 8.

Next, we considered the actual mixed signal $s(t)$ with additive Gaussian white noise of different amplitudes according to (21) in an SNR range from $-15 \mathrm{~dB}$ to $5 \mathrm{~dB}$. Here, EEMD-GST, ST, and STFT were repeated in simulation for 100 times, and Monte Carlo experiments were conducted for 6,300 times in total using an equivalent simulation platform as that employed in the previous subsection. The simulations generated 6,300 RGB images with a pixel ratio of $672 \times$ 503 , and the time-frequency distribution results for EEMDGST, ST, and STFT under an SNR of $-12 \mathrm{~dB}$ are shown in Figures 9(a), 9(b), and 9(c), respectively. A comparison of the three figures indicates that EEMD-GST retains reasonable signal characteristics under a strong noise background, while ST and STFT are more strongly influenced by noise.
As can be seen intuitively from Figure 9, the method proposed in this paper has significantly improved efficiency compared with the classical algorithm.

To quantitatively evaluate the relative performances of the three algorithms, we take Figure 8(a) as the standard timefrequency distribution image and measure algorithm performance by calculating the peak SNR (PSNR) $[28,29]$ and the structural similarity (SSIM) $[30,31]$ based on comparisons of the images generated under a noise background of varying SNR.

PSNR represents the ratio between the maximum possible power of a signal and the power of destructive noise that affects its expression accuracy. It is commonly used as a measurement method for signal reconstruction quality in image compression and other fields. Structural similarity is an index to measure the similarity of two images. SSIM index describes structural information as independent of brightness and contrast from the perspective of image composition, which reflects the properties of object structure in the scene. 


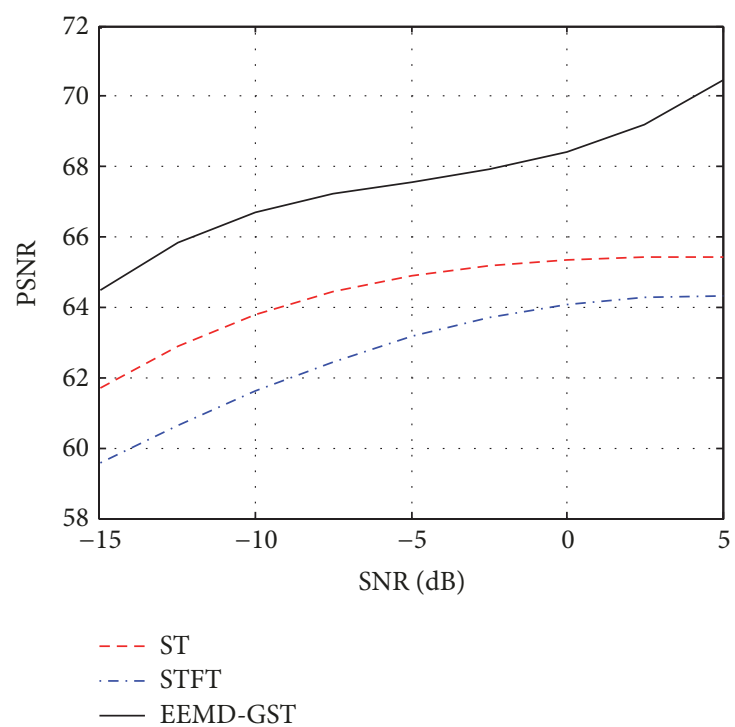

(a) PSNR comparison diagram under different signal-to-noise ratios

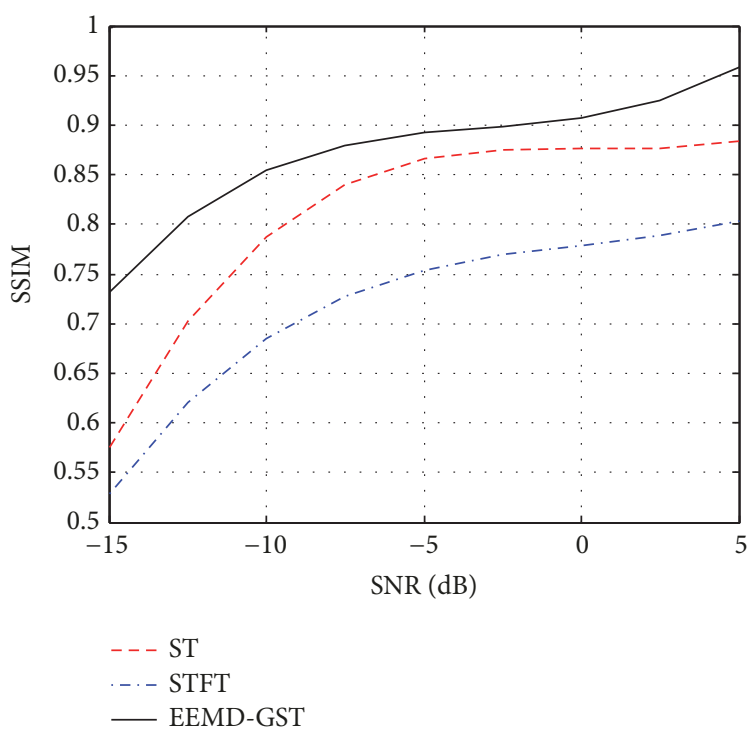

(b) SSIM comparison diagrams under different signal-to-noise ratios

FIGURE 10: Comparison of the (a) peak SNR (PSNR) and (b) structural similarity (SSIM).

It has become a widely used method to measure video quality in broadcast and cable TV.

In this paper, PSNR is used to measure the noise resistance of the algorithm, and SSIM is used to quantitatively describe the algorithm's noise suppression and image similarity after restoration. PSNR and SSIM are used to measure the antinoise performance of the algorithm.

To guarantee the credibility of our simulation results, simulation verification was conducted using the MATLAB source code written by Ronna Fattal. The PSNR and SSIM results obtained are given in Figures 10(a) and 10(b), respectively. From Figure 10(a), we note that the PSNR obtained by EEMD-GST is always greater than those obtained by ST and STFT. Meanwhile, the PSNR values obtained by ST and STFT approach a stable maximum for an SNR greater than $0 \mathrm{~dB}$, but the PSNR values obtained by EEMD-GST continue increasing toward a much higher stable maximum. Similar results are observed for the SSIM values shown in Figure 10(b), where EEMD-GST provides greater SSIM values over all SNR values considered, particularly for relatively low SNR values below $-5 \mathrm{~dB}$.

The simulation and theoretical analyses conducted in previous subsections demonstrate that EEMD has relatively strong antinoise performance. Therefore, the EEMD-GST approach can obtain more ideal data preprocessing under low SNR conditions. Meanwhile, compared to ST and STFT, the GST demonstrates a better time-frequency focusing property. Thus, radar signal characteristics are more clearly represented, and the EEMD-GST approach can be expected to be more effective for recognizing the specific modulation of radar signal emitters. However, the order of the computational complexity of the signal analyses considered is $\sigma(\mathrm{GST})>\sigma(\mathrm{ST})>\sigma(\mathrm{STFT})$ because the proposed EEMDGST approach conducts GST on each IMF after applying
EEMD to radar signals. Therefore, the recognition speed associated with the EEMD-GST approach must be relatively low. Under the present conditions of rapid radar technology development, the adoption of advanced algorithms offering improved emitter recognition performance is advisable, even if those algorithms offer somewhat reduced recognition speed, because improved computational performance can be expected in the future.

In order to further reflect the performance of this method, literature [32] in the EEMD-ICA (Ensemble Empirical Mode Decomposition-Independent Component Analysis), literature [33] in the CEEMD (Complete Ensemble Empirical Mode Decomposition), and literature [34] in EEMD-PCA (Ensemble Empirical Mode Decomposition- Principal Component Analysis) algorithm were also based on SSIM for comparison. The algorithms in literature [32-34] have not involved time-frequency analysis; however, in literature [32], the SSIM of the recovered signal and the original signal after calculation is used to measure the performance of the algorithm. By referring to its idea, the SSIM is used to measure the ability of the algorithm to fight against noise. The simulation conditions and environment are the same as above, and SSIM diagrams of the three algorithms are shown in Figure 11.

It can be seen from Figure 11 that the EEMD-GST algorithm in this paper is slightly stronger than the other three methods in SSIM; in particular, under the condition of low SNR, the method in this paper achieves better results. The reason is that EEMD can better weaken the influence of noise, and the generalized S-transform can also play a role in noise suppression. EEMD-ICA method for signal independent demand is higher, the higher the independence between signals, the strength of the algorithm efficiency, more dependent on the signal between the independence 


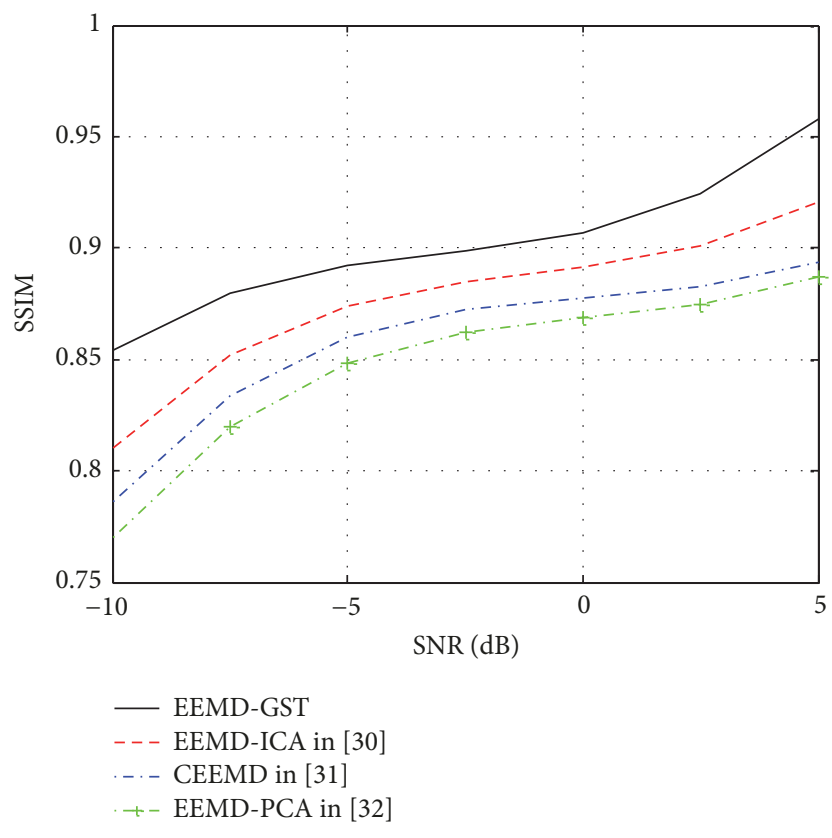

FIGURE 11: Comparison of the structural similarity with other algorithms (SSIM).

between signal and noise, and simulation, this paper does not require between each source, the source and the noise is independent, thus effect is weaker than the method, and fall, but the independence between signals, method of limited effectiveness. Compared with EEMD, CEEMD has better processing effect. However, EEMD is more stable and mature than CEEMD, and it is more convenient to process. Due to the parameter adaptive adjustment system constructed in this paper, the parameters of the generalized S-transform can be more matched with the signal to be detected. And the GST algorithm itself has the ability to reduce noise and improve signal characteristics. Therefore, the EEMD-GST algorithm overall performance is stronger than CEEMD. It is suitable for the recognition of signals. EEMD-PCA, in the process of PCA processing, has lost a small part of the original signal information, especially with the reduction of signal-to-noise ratio, this loss gradually increases, so in the condition of low signal-to-noise ratio, its algorithm efficiency compared to the algorithm in this paper, performance gap.

To sum up, compared with the classical algorithm and the current mainstream improved algorithm, the algorithm in this paper has improved in efficiency, and the result is more intuitive. It is also more accurate and has good applicability for processing the signal with low signal-to-noise ratio.

\section{Conclusions}

Present radar signal emitter recognition approaches suffer most profoundly from a severe dependency on prior information. Moreover, the development of emitter recognition methods must meet the challenges associated with the present and future development of LPI technology and other obscuration methodologies based on complex signal modulation. Simultaneously, modern radar reconnaissance interception receivers are required to provide a relatively strong ability to extract weak signals under low SNR values. Therefore, the present study eliminated this dependency on prior information by combining EEMD with the GST to realize the robust recognition of radar signal emitters. The basic theories of EMD, EEMD, and GST were presented, and the procedure for conducting EEMD-GST was defined. Monte Carlo simulations were conducted to demonstrate the advantages of the proposed EEMD-GST approach from three different perspectives. Firstly, the decomposition results obtained with EMD and EEMD were compared when applied to a mixed signal formed as a linear superposition of a highfrequency intermittent signal and a simple continuous signal. Secondly, EMD and EEMD were applied to decompose mixed signals composed of a standard LFM signal and additive Gaussian white noise of different amplitudes. Thirdly, EEMDGST processing was applied to a mixed signal involving the linear superposition of several typical radar signals with additive white Gaussian noise, and the analysis results obtained were compared with the corresponding results obtained using the standard ST and the STFT. The results of these simulations can be outlined as follows:

(1) For the linear superposition of a high-frequency intermittent signal and a simple continuous signal, EEMD was demonstrated to effectively suppress mode mixing.

(2) For the mixed signals composed of a standard LFM signal and additive Gaussian white noise of different amplitudes, EEMD extracted meaningful signal features even under low SNR values, which demonstrated its ability to suppress noise.

(3) For the mixed signals composed of several typical radar signals with additive white Gaussian noise of different amplitudes, EEMD-GST provided an obviously better timefrequency focusing property than that of either ST or STFT. In addition, the PSNR and SSIM values obtained by EEMDGST were always greater than those obtained by ST and STFT, particularly for relatively low SNR values.

While the present work has provided an integrated and improved radar signal emitter recognition approach, the mathematical expression of the signal after conducting EEMD-GST remains to be researched in detail to enable the quantitative characterization of emitter recognition performance and provide a basis for further research.

\section{Data Availability}

The data used to support the findings of this study are included within the article.

\section{Conflicts of Interest}

The authors declare that there are no conflicts of interest regarding the publication of this paper.

\section{Acknowledgments}

This work was supported in part by the National Natural Science Foundation of China under Grant 67103427, 61502522, 
61472442, and 61472443 and Natural Science Foundation of Shaanxi Province under 2017JQ6035 and 2016JM6017.

\section{References}

[1] M.-W. Liu and J. F. Doherty, "Nonlinearity estimation for specific emitter identification in multipath channels," IEEE Transactions on Information Forensics and Security, vol. 6, no. 3, pp. 1076-1085, 2011.

[2] A. E. Spezio, "Electronic warfare systems," IEEE Transactions on Microwave Theory and Techniques, vol. 50, no. 3, pp. 633-644, 2002.

[3] G. Latombe, E. Granger, and F. A. Dilkes, "Fast learning of grammar production probabilities in radar electronic support," IEEE Transactions on Aerospace and Electronic Systems, vol. 46, no. 3, pp. 1037-1041, 2010.

[4] H. J. Liu, Z. Liu, W. L. Jiang et al., "Approach based on cloud model and vector neural network for emitter identificationl," Acta Electronica Sinica, vol. 38, no. 12, pp. 2797-2804, 2010.

[5] Q. Guo and Y. He, "DSm evidence modeling and radar emitter fusion recognition method based on cloud model," Journal of Electronics \& Information Technology, vol. 37, no. 8, pp. 17791785, 2015.

[6] X. Guan, G. D. Sun, Q. Guo et al., "Radar emitter parameter recognition based on interval number and evidence theory," Systems Engineering and Electronics, vol. 36, no. 7, pp.1269-1274, 2014.

[7] L. Wang, L. N. Zhou, H. B. Ji et al., "A new matching pursuit algorithm for signal classification," Journal of Electronics \& Information Technology, vol. 36, no. 6, pp. 1299-1306, 2014.

[8] A. Wang and V. Krishnamurthy, "Signal interpretation of multifunction radars: modeling and statistical signal processing with stochastic context free grammar," IEEE Transactions on Signal Processing, vol. 56, no. 3, pp. 1106-1119, 2008.

[9] H. J. Liu, Y. Li, Z. Liu et al., "Approach to multi-function radar identification based on stochastic grammars," Acta Aeronautica et Astronautica Sinica, vol. 31, no. 9, pp. 1809-1817, 2010.

[10] S. Ma, Z. Liu, and W. L. Jiang, "The application of gene techniques to multifunction radar signal analysis," Acta Electronica Sinica, vol. 41, no. 12, pp. 2374-2381, 2013.

[11] J.-P. Kauppi, K. Martikainen, and U. Ruotsalainen, "Hierarchical classification of dynamically varying radar pulse repetition interval modulation patterns," Neural Networks, vol. 23, no. 10, pp. 1226-1237, 2010.

[12] G. P. Kuang, J. G. Wang, and J. Q. Luo, "Radar emitter recognition based on pulse sequence pattern and Vague set," Journal of Astronautics, vol. 32, pp. 1639-1644, 2011.

[13] C. W. Qu, N. Li, and F. Su, "Emitter gray identification based on variable precision rough set model," Control \& Decision, vol. 26, no. 5, pp. 753-757, 2011.

[14] X. Guan, Y. He, and X. Yi, "A novel rough set emitter signal recognition model," Journal of Astronautics, vol. 28, no. 3, pp. 685-688, 2007.

[15] O. A. Olvera-Guerrero, A. Prieto-Guerrero, and G. EspinosaParedes, "Non-linear stability analysis of real signals from nuclear power plants (boiling water reactors) based on noise assisted empirical mode decomposition variants and the shannon entropy," Entropy, vol. 19, no. 7, pp. 1-33, 2017.

[16] Y. Luo, Y. Q. Bai, and Q. Zhang, "Translational motion compensation and micro-doppler feature extraction of ballistic targets,"
Journal of Electronics Information Technology, vol. 34, no. 3, pp. 602-608, 2012.

[17] X. Hao, Q. Liu, G. Yang, and Y. Du, "Using the EMD method to determine fault criterion for medium-low pressure gas regulators," Journal of Thermal Science, vol. 24, no. 6, pp. 557561, 2015.

[18] O. A. Olvera-Guerrero, A. Prieto-Guerrero, and G. EspinosaParedes, "Decay Ratio estimation in BWRs based on the improved complete ensemble empirical mode decomposition with adaptive noise," Annals of Nuclear Energy, vol. 102, pp. 280296, 2017.

[19] H. Wang, Z. J. Hu, Z. Chen et al., "A hybrid model for wind power forecasting based on ensemble empirical mode decomposition and wavelet neural networks," Transactions of China Electrotechnical Society, vol. 28, no. 9, pp. 137-141, 2013.

[20] Z. H. Wu and N. E. Huang, "Ensemble empirical mode decomposition: a noise-assisted data analysis method," Advances in Adaptive Data Analysis (AADA), vol. 1, no. 1, pp. 1-41, 2009.

[21] Z. Wu, N. E. Huang, S. R. Long, and C. Peng, "On the trend, detrending, and the variability of nonlinear and nonstationary time series," Proceedings of the National Acadamy of Sciences of the United States of America, vol. 104, no. 38, pp. 14889-14894, 2007.

[22] D. S. Shan, Q. Li, and Z. Huang, "Adaptive decomposition and reconstruction for bridge structural dynamic testing signals," Journal of Vibration and Shock, vol. 34, no. 3, pp. 1-7, 2015.

[23] X. Liu, W. Huang, and E. W. Gill, "Wind direction estimation from rain-contaminated marine radar data using the ensemble empirical mode decomposition method," IEEE Transactions on Geoscience and Remote Sensing, vol. 55, no. 3, pp. 1833-1841, 2017.

[24] Z. Wei, K. G. Robbersmyr, and H. R. Karimi, "An EEMD aided comparison of time histories and its application in vehicle safety," IEEE Access, vol. 5, pp. 519-528, 2017.

[25] E. Sejdi, I. Djurovi, and J. Jiang, "A window width optimization S-transform," Journal on Advances in Signal Processing, vol. 1, Article ID 672941, 2008.

[26] I. Djurović, E. Sejdić, and J. Jiang, "Frequency-based window width optimization for S-transform," AEÜ - International Journal of Electronics and Communications, vol. 62, no. 4, pp. 245250, 2008.

[27] L. Stanković, "Measure of some time-frequency distributions concentration," Signal Processing, vol. 81, no. 3, pp. 621-631, 2001.

[28] S.-M. Tseng and Y.-F. Chen, "Average PSNR optimized cross layer user grouping and resource allocation for uplink MUMIMO OFDMA video communications," IEEE Access, vol. 6, pp. 50559-50571, 2018.

[29] H. Yuan, S. Kwong, X. Wang, Y. Zhang, and F. Li, "A virtual view PSNR estimation method for 3-D Videos," IEEE Transactions on Broadcasting, vol. 62, no. 1, pp. 134-140, 2016.

[30] W. Gao, S. Kwong, Y. Zhou, and H. Yuan, "SSIM-based game theory approach for rate-distortion optimized intra frame CTU-level bit allocation," IEEE Transactions on Multimedia, vol. 18, no. 6, pp. 988-999, 2016.

[31] T. Zhao, J. Wang, Z. Wang, and C. W. Chen, "SSIM-based coarse-grain scalable video coding," IEEE Transactions on Broadcasting, vol. 61, no. 2, pp. 210-221, 2015.

[32] K. Zeng, D. Chen, G. Ouyang, L. Wang, X. Liu, and X. Li, “An EEMD-ICA approach to enhancing artifact rejection for noisy multivariate neural data," IEEE Transactions on Neural Systems and Rehabilitation Engineering, vol. 24, no. 6, pp. 630-638, 2016. 
[33] J. Li, C. Liu, Z. Zeng, and L. Chen, "GPR signal denoising and target extraction with the CEEMD method," IEEE Geoscience and Remote Sensing Letters, vol. 12, no. 8, pp. 1615-1619, 2015.

[34] M. A. Motin, C. K. Karmakar, and M. Palaniswami, "Ensemble empirical mode decomposition with principal component analysis: a novel approach for extracting respiratory rate and heart rate from photoplethysmographic signal," IEEE Journal of Biomedical and Health Informatics, vol. 22, no. 3, pp. 766-774, 2018. 


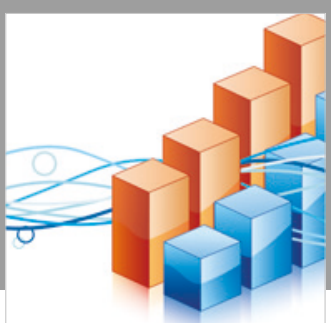

Advances in

Operations Research

\section{-n-m}
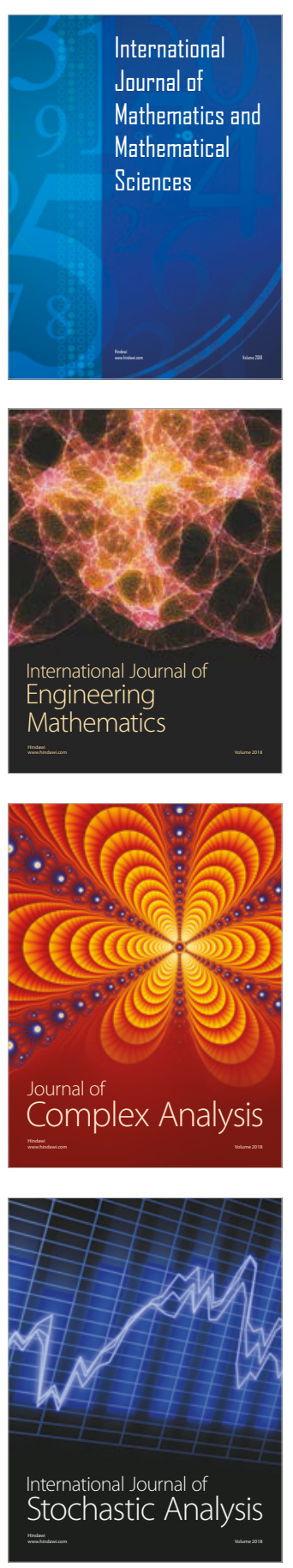
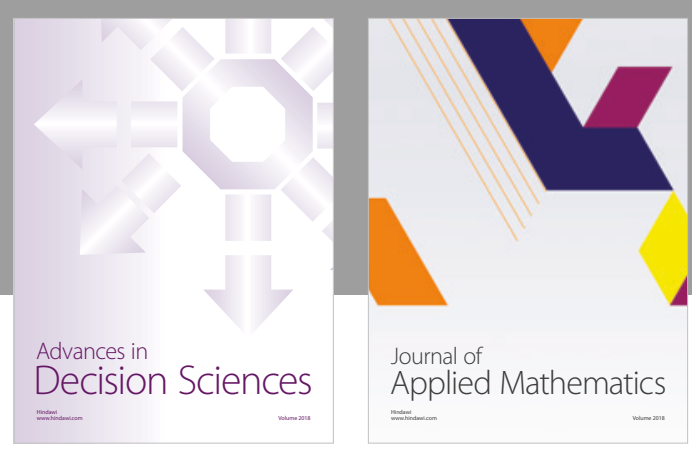

Journal of

Applied Mathematics
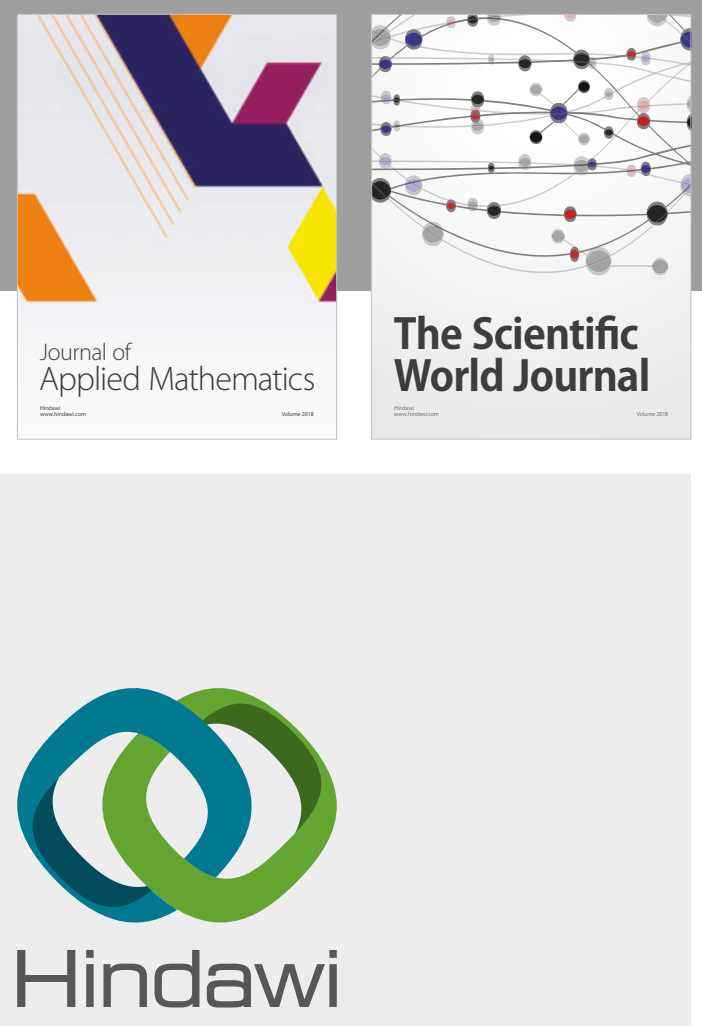

Submit your manuscripts at

www.hindawi.com

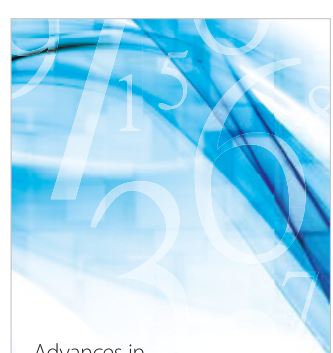

Advances in
Numerical Analysis
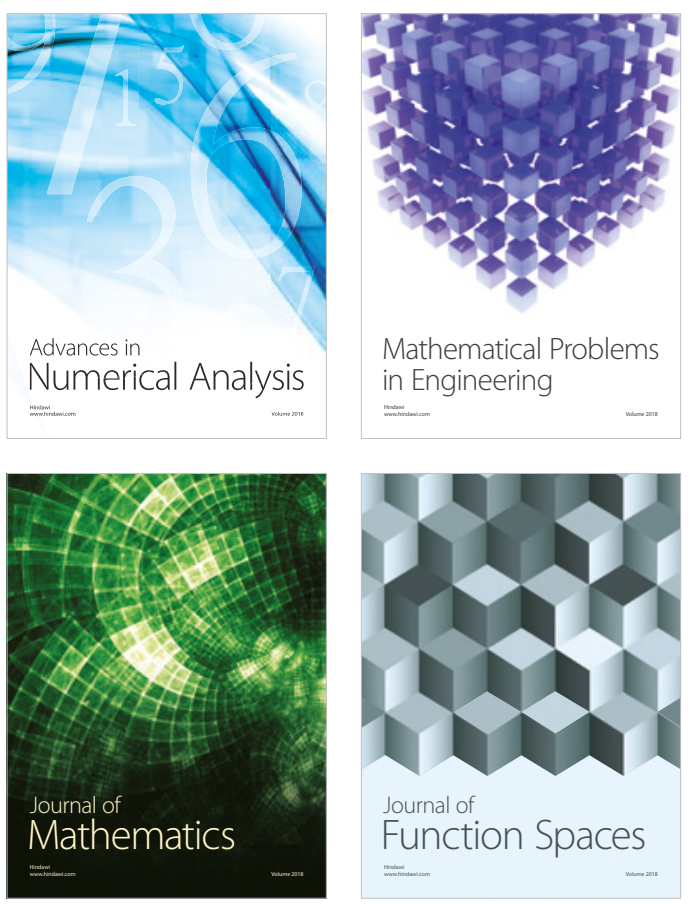

Mathematical Problems in Engineering

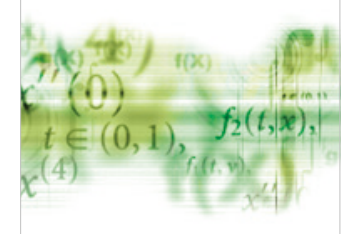

International Journal of

Differential Equations

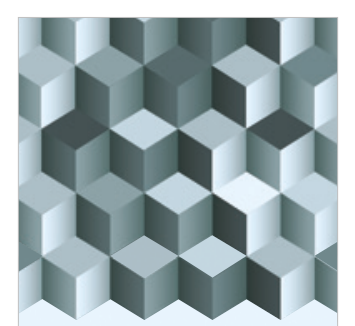

Journal of

Function Spaces

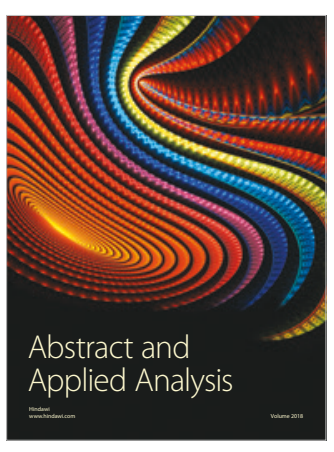

The Scientific

World Journal

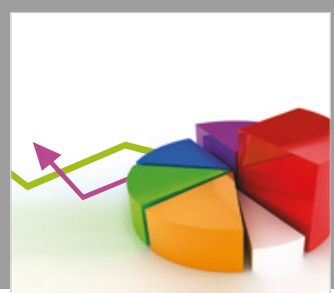

Journal of

Probability and Statistics
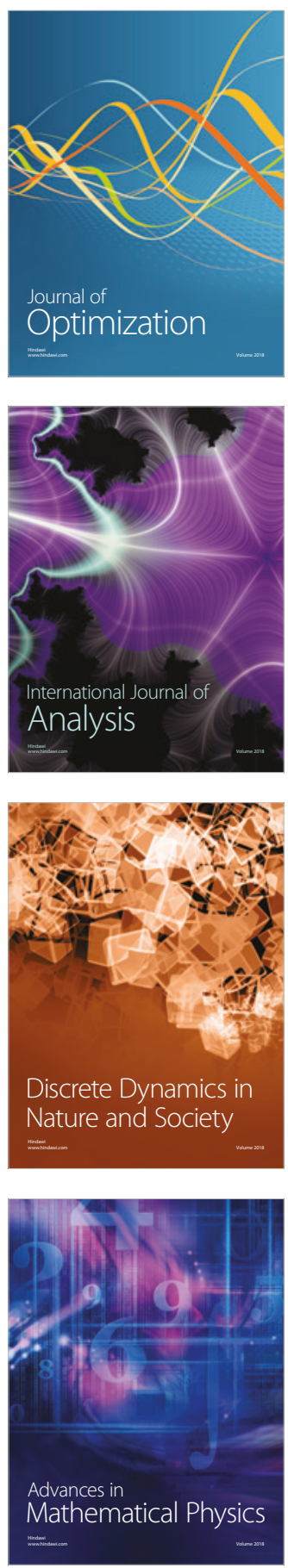